\title{
Myocardial salvage by T2W-CMR: direct comparison to a non-destructive, high resolution, 3-dimensional ex-vivo assessment of the area at risk simultaneous with infarction
}

\author{
Lowie M Van Assche*, Christoph J Jensen, David Wendell, Michele Parker, Han W Kim, Raymond J Kim \\ From 16th Annual SCMR Scientific Sessions \\ San Francisco, CA, USA. 31 January - 3 February 2013
}

\section{Background}

The amount of myocardial salvage is a critical determinant of prognosis in acute myocardial infarction (AMI). T2WCMR in combination with DE-CMR is thought to be a promising method that could presumably measure salvage. However, direct comparisons with the appropriate reference standard are limited. Additionally, even if a pathology reference standard is present, co-registration with in-vivo imaging is problematic. We recently validated a new ex-vivo CMR protocol against the reference standard of microspheres and TTC that provides 3D, non-destructive, high-resolution maps of the AAR simultaneously with infarction[1]. Because this map delineates both the AAR and infarction, salvage can be easily measured in a single image, rather than being calculated from 2 datasets. Additionally, the 3D map allows for direct matching of in-vivo DE to ex-vivo CMR and thereby further minimizing the potential for misregistration from in-vivo $\mathrm{DE}$ and more importantly in-vivo T2. Therefore, we sought to compare in-vivo calculated salvage to the directly measured salvage by ex-vivo CMR.

\section{Methods}

12 canines underwent variable coronary occlusion of the LAD (45-75min) followed by reperfusion to create a range of myocardial salvage. CMR was performed 5-days post-AMI. T2W-CMR was performed using an optimized DIR-TSE sequence with reduced inter-echo spacing and significantly improved image homogeneity [2]. Following in-vivo imaging, the $3 \mathrm{D}$ ex-vivo protocol was performed delineating viable AAR (dark), infarcted AAR (bright) and normal myocardium (grey). Ex-vivo CMR slices were directly matched to in-vivo DE images. In-vivo salvage was calculated by subtracting infarct size by DE from T2hyperintensity size by TSE and expressed as a percentage of T2-hyperintensity size. For ex-vivo CMR, salvage was directly measured on the matched slices and expressed as a percentage of the AAR.

\section{Results}

A total of 51 slices were analyzed. Mean calculated salvage by in-vivo CMR was significantly lower then mean measured salvage by ex-vivo CMR. (3.2\% vs $63.6 \%$, respectively, $\mathrm{p}<0.0001)$. Figure 1 shows no correlation between in-vivo and ex-vivo CMR ( $\mathrm{r}=-0.08, \mathrm{p}=0.55)$. Conversely, there was a significant correlation between $\mathrm{T} 2$ size by in-vivo CMR and scar size by the ex-vivo protocol $(r=0.93, p<0.0001)$. Figure 2 shows examples of in-vivo T2 and DE slices compared with directly marched ex-vivo CMR.

\section{Conclusions}

In-vivo calculated salvage did not correspond with directly measured salvage on matched ex-vivo slices. Instead, there was a strong correlation between in-vivo $\mathrm{T} 2$ size and ex-vivo scar size.

\section{Funding}

Funded in part by 5R01HL064726-07. 


\section{Figure 1}

A)

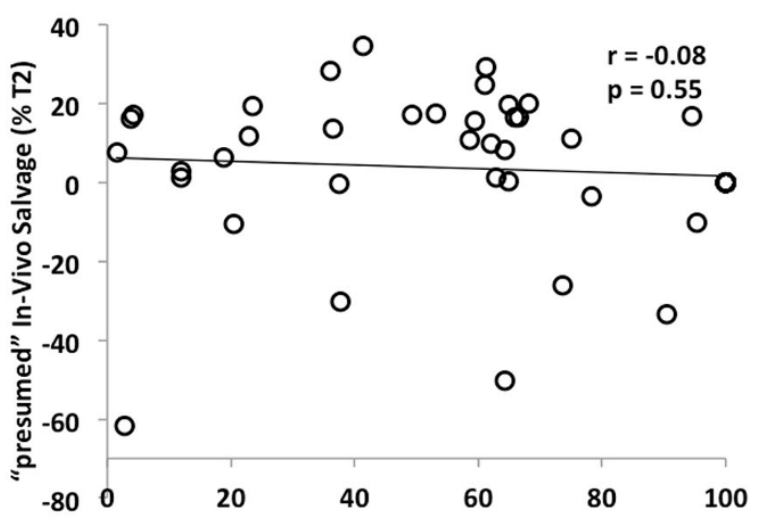

"True" Ex-Vivo Salvage (\% AAR)

B)

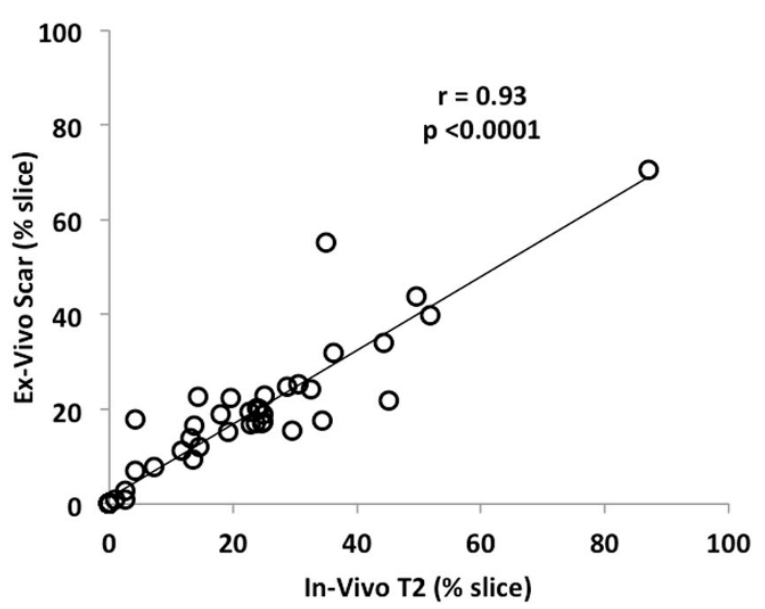

Figure 1

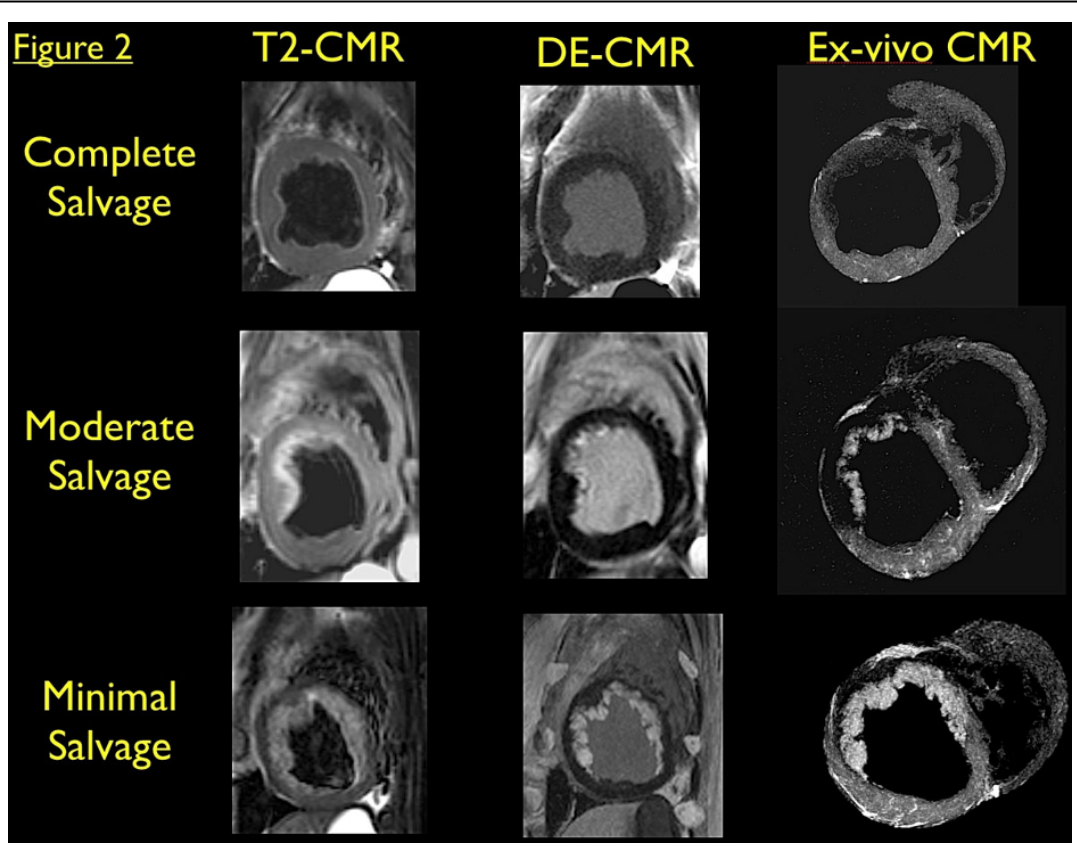


Published: 30 January 2013

\section{References}

1. JCMR 2012, 14(1), Abstract O7.

2. JCMR 2012, 14(1), Abstract O113.

\section{doi:10.1186/1532-429X-15-S1-015}

Cite this article as: Van Assche et al: Myocardial salvage by T2W-CMR: direct comparison to a non-destructive, high resolution, 3-dimensional ex-vivo assessment of the area at risk simultaneous with infarction. Journal of Cardiovascular Magnetic Resonance 2013 15(Suppl 1):015.

Submit your next manuscript to BioMed Central and take full advantage of:

- Convenient online submission

- Thorough peer review

- No space constraints or color figure charges

- Immediate publication on acceptance

- Inclusion in PubMed, CAS, Scopus and Google Scholar

- Research which is freely available for redistribution

Submit your manuscript at www.biomedcentral.com/submit
() Biomed Central 